\title{
AN APPROACH TO EXPONENTIATION WITH INTERVAL-VALUED POWER
}

\author{
Anna Tikhonenko-Kędziak, Miroslaw Kurkowski \\ Faculty of Mathematics and Natural Sciences, College of Sciences \\ Cardinal Stefan Wyszynski University in Warsaw \\ Warsaw, Poland \\ a.tikhonenko@uksw.edu.pl,m.kurkowski@uksw.edu.pl
}

Received: 21 October 2016; accepted: 21 November 2016

\begin{abstract}
The main aim of the work is introducing an operation of raising intuitionistic fuzzy values to intuitionistic fuzzy power, which not requiring to conversion of intuitionistic fuzzy values. Introducing an operation of raising intuitionistic fuzzy values to intuitionistic fuzzy power, which does not require conversion of intuitionistic fuzzy values is the main aim of the work. It is known that, in the classical intuitionistic fuzzy sets theory, the use of all aggregation modes is not always possible because of the lack of definition of raising intuitionistic fuzzy values to intuitionistic fuzzy power. Therefore, the specific aim of the work is to present the heuristic method of raising intuitionistic fuzzy values to intuitionistic fuzzy power, and the consideration of its properties.
\end{abstract}

Keywords: IVFS, DST, Dempster-Shafer theory, interval-values fuzzy sets, operations on IVFS's

\section{Introduction}

It is known that different variants of the aggregation of local criteria give rise to different results. It follows from this fact that the validity of the stage of formulation of a global criterion as an aggregation of local criteria is dominant. It is obvious that the evaluation of the validity of the criteria is not essential in some optimization processes and sometimes all local criteria have the same validity (weight) for decision-makers. What's more, it is sometimes impossible to define the weight by using real numbers. Therefore, it is more proper to use the transformation of verbal terms to interval or fuzzy values applied to various types of aggregation modes. Many aggregation modes are available to use to make decisions. Not only real numbers [1-12] can be used to describe them.

One of the most common generalizations of the fuzzy sets theory is the one introduced by Atanassov [3], which is mainly used for solving MCDM [13-19] and group MCDM [5, 6, 20-25] problems in cases when intuitionistic fuzzy values are the value of the local criteria of alternatives and/or their weight. 
Disadvantages of classical operations on intuitionistic fuzzy values are the base of some issues with intuitionistic fuzzy uncertainty in MCDM framework. A few limitations of traditional operations on fuzzy values were conferred in [26]. In [27], appropriate critical examples can be read about.

An approach based on the intuitionistic fuzzy matrix and the relations between the elements of the matrix, or the intuitionistic fuzzy sets, were introduced in [28]. In [27] it was confirmed that correct results cannot always be obtained by the method of comparing the intuitionistic fuzzy numbers introduced in [28].

The lack of a definition of raising intuitionistic fuzzy values to intuitionistic fuzzy power is another issue which can be experienced with the classical intuitionistic fuzzy sets theory. It is noteworthy that the lack of such a definition severely curtails the number of aggregation modes which can be applied in MCDM problems under the circumstances of fuzzy uncertainty.

In paper [27], it was demonstrated that the use of the Dempster-Shafer theory based on converting intuitionistic fuzzy values to belief intervals allows one to achieve results which are more reliable, as well as facilitates the calculations in the solution of the MCDM problem. However, when using the conversion of the intuitionistic fuzzy values is not appropriate, using the heuristic method of raising intuitionistic fuzzy values to intuitionistic fuzzy power is advisable.

The remaining part of the paper is organized in the following way. The basic definitions of the classical intuitionistic fuzzy sets theory are given in section 2 . In section 3, we present the extension of the intuitionistic fuzzy set theory in the framework of the Dempster-Shafer theory. Section 4 is devoted to an operation on the intuitionistic fuzzy sets in the framework of the Dempster-Shafer theory, and new operators of raising intuitionistic fuzzy values to intuitionistic fuzzy power, as well as the Intuitionistic Fuzzy Weighted Geometric operator with weights presented by intuitionistic fuzzy values are introduced. In section 5 , a few properties of the exponentiation operation achieved by the transformation to belief intervals and the heuristic IFV method were proved.

The study is cofounded by the European Union from resources of the European Social Fund. Project PO KL „Information technologies: Research and their interdisciplinary applications", Agreement UDA-POKL.04.01.01-00-051/10-00.

\section{Basic definitions}

One of the most common generalizations of the fuzzy sets is the intuitionistic fuzzy sets theory which was introduced by Atanassov [3] which is mainly used for solving issues with MCDM [7, 14-19] and group MCDM [5, 6, 20-25] under the circumstances when the intuitionistic fuzzy values are the value of the local criteria of alternatives and/or their weight.

The base of the definition of the intuitionistic fuzzy set are the considerations of membership function $\mu$ and non-membership function $v$ of element $x$ to a set $X$, where $0 \leq \mu(x)+v(x) \leq 1$. After that, the following set can be constructed 
$\{\langle x, \mu(x), v(x)\rangle \mid x \in E\}$, where $0 \leq v(x)+\mu(x) \leq 1$. For constant $x \in E$, pair $\langle\mu(x), v(x)\rangle$ is called the intuitionistic fuzzy value (IFV) or the intuitionistic fuzzy number.

A few operators based on the synthesis of intuitionistic fuzzy sets and the Dempster-Shafer Theory (DST) were presented in sections [29] and [30]. It can easily be seen that the operators which base on the Choquet integral [30] are advantageous under circumstances where the aggregate weight of the assessments have some correlation with one other.

The strong connection between the intuitionistic fuzzy sets and DST was presented in [31]. This connection allows one to directly apply Dempster's rule of combination in MCDM issues in order to aggregate local criteria with intuitionistic fuzzy values. This connection, or link, was also presented in $[32,33]$.

In [3] Atanassov gives the following definition of intuitionistic fuzzy set:

Definition 1. Let $X=\left\{x_{1}, x_{2}, \ldots, x_{n}\right\}$ be finite universal set. An object $A$ in $X$ is called intuitionistic fuzzy set (IFS) if it has the following form: $A=\left\{\left\langle x_{j}, \mu_{A}\left(x_{j}\right), v_{A}\left(x_{j}\right)\right\rangle \mid x_{j} \in X\right\}, \quad$ where functions $\mu_{A}: X \rightarrow[0,1]$, $x_{j} \in X \rightarrow \mu_{A}\left(x_{j}\right) \in[0,1]$ and $v_{A}: X \rightarrow[0,1], x_{j} \in X \rightarrow v_{A}\left(x_{j}\right) \in[0,1]$ determines the degree of membership and non-membership of element $x_{j} \in X$ of $A \subseteq X$ respectively, and, for each $x_{j} \in X$, the inequality $0 \leq \mu_{A}\left(x_{j}\right)+v_{A}\left(x_{j}\right) \leq 1$ holds.

Parameter $\pi_{A}(x)=1-\mu_{A}\left(x_{j}\right)-v_{A}\left(x_{j}\right)$ is called an intuitionistic index (or the hesitation degree) of the element $x_{j}$ in $A$ [3].

Of course, for each $x_{j} \in X$, we have $0 \leq \pi_{A}(x) \leq 1$.

Being that an intuitionistic set is the generalization of a typical fuzzy one, all of the regular results from the classical fuzzy set theory may be converted in the framework of the intuitionistic fuzzy sets theory (IFST), and all of the studies which base on regular fuzzy sets can be expressed by IFSs. However, IFST does not only contain operations which are compatible on fuzzy sets, but also such operations which cannot be expressed in the framework of the regular fuzzy set theory [34].

The operations of addition $\oplus$ and multiplication $\otimes$ on IFV are defined by Atanassov [4] as follows. Let $A=\left\langle\mu_{A}, v_{A}\right\rangle$ and $B=\left\langle\mu_{B}, v_{B}\right\rangle$ be IFV's. Then

$$
\begin{aligned}
& A \oplus B=\left\langle\mu_{A}+\mu_{B}-\mu_{A} \mu_{B}, v_{A} v_{B}\right\rangle, \\
& A \otimes B=\left\langle\mu_{A} \mu_{B}, v_{A}+v_{B}-v_{A} v_{B}\right\rangle .
\end{aligned}
$$

These operations were constructed in such a way that the result of their use is IFV. It is easy to prove that $0 \leq \mu_{A}+\mu_{B}-\mu_{A} \mu_{B} \leq 1$ and $0 \leq v_{A}+v_{B}-v_{A} v_{B} \leq 1$.

Based on operations (1) and (2) the following expressions were received in [35] for each integer $n=1,2, \ldots$ : 


$$
n A=A \oplus \ldots \oplus A=\left\langle 1-\left(1-\mu_{A}\right)^{n}, v_{A}{ }^{n}\right\rangle, A^{n}=A \otimes \ldots \otimes A=\left\langle\mu_{A}{ }^{n}, 1-\left(1-v_{A}{ }^{n}\right)\right\rangle .
$$

It was shown that these operations can be used not only for integer values, but also for the real values $\lambda>0$, i.e.:

$$
\begin{aligned}
& \lambda A=\left\langle 1-\left(1-\mu_{A}\right)^{\lambda}, v_{A}^{\lambda}\right\rangle, \\
& A^{\lambda}=\left\langle\mu_{A}^{\lambda}, 1-\left(1-v_{A}{ }^{\lambda}\right)\right\rangle .
\end{aligned}
$$

The operations (1)-(4) have the following algebraic properties [36]:

Let $A=\left\langle\mu_{A}, v_{A}\right\rangle$ and $B=\left\langle\mu_{B}, v_{B}\right\rangle$ be IFV's. Then

$$
\begin{gathered}
A \oplus B=B \oplus A, \\
A \otimes B=B \otimes A, \\
\lambda(A \oplus B)=\lambda A \oplus \lambda B, \\
(A \otimes B)^{\lambda}=A^{\lambda} \otimes B^{\lambda}, \\
\lambda_{1} A \oplus \lambda_{2} A=\left(\lambda_{1} \oplus \lambda_{2}\right) A, \lambda_{1}, \lambda_{2}>0, \\
A^{\lambda_{1}} \otimes A^{\lambda_{2}}=A^{\lambda_{1}+\lambda_{2}}, \lambda_{1}, \lambda_{2}>0 .
\end{gathered}
$$

Operations (1)-(4) are used for aggregation of local criteria in the case of solving the MCDM problems in terms of fuzzy intuitionistic uncertainty.

Let $A_{1}, \ldots, A_{n}$ be an IFV's of local criteria and $w_{1}, \ldots, w_{n}\left(\sum_{i=1}^{n} w_{1}=1\right)$ be a weight of this criteria. Then the Intuitionistic Weighted Arithmetic Mean (IWAM) may be specified by using the operation (1) and (3) as follows [31]:

$$
I W A M=w_{1} A_{1} \oplus w_{2} A_{2} \oplus \ldots \oplus w_{n} A_{n}=\left\langle 1-\prod_{i=1}^{n}\left(1-\mu_{A_{i}}\right)^{w_{i}}, \prod_{i=1}^{n} v_{A_{i}}{ }^{w_{i}}\right\rangle .
$$

The result is obtained by the aggregation operator (11) in the IFV for and its idempotent. It is this aggregation operator that is the most common in MCDM problem solving under the circumstances of intuitionistic fuzzy uncertainty.

It is also worth noting that there are no problems with the idempotent Intuitionistic Fuzzy Weighted Geometric operator (IFWG), which can be obtained directly from (2) and (4):

$$
I F W G=A_{1}^{w_{i}} \otimes A_{2}^{w_{i}} \otimes \ldots \otimes A_{n}^{w_{i}}=\left\langle\prod_{i=1}^{n} \mu_{A_{i}}^{w_{i}}, 1-\prod_{i=1}^{n}\left(1-v_{A_{i}}\right)^{w_{i}}\right\rangle .
$$




\section{An extension of intuitionistic fuzzy set theory in the framework of DST}

The close connection between the intuitionistic fuzzy sets and DST was proved in paper [31]. This connection allows one to directly apply the Dempster's rule of combination in MCDM issues in order to aggregate local criteria with intuitionistic fuzzy values.

Furthermore, in paper [31], the possibility of transforming intuitionistic fuzzy values to Belief Intervals (BI), based on the extension of intuitionistic fuzzy sets theory in the context of DST was also presented. The presentation of mathematical operations on the IFVs as operations on BI is allowed by this fact.

In [37] Shafer introduced several measures. The belief measure is a mapping $\mathrm{Bel}: 2^{X} \rightarrow[0,1]$, such that for any subset $B$ from $X$ occurring the expression [31]:

$$
\operatorname{Bel}(B)=\sum_{i=1}^{n} m\left(A_{i}\right), A_{i} \subseteq B, i=1 \text { to } n .
$$

The next measure proposed by Shafer is a measure of plausibility, which is a mapping $P l: 2^{X} \rightarrow[0,1]$, such that for any subset $B$ from $X$ the relation [31]

$$
P l(B)=\sum_{i=1}^{n} m\left(A_{i}\right), A_{i} \cap B, i=1 \text { to } n .
$$

holds.

It can easily be seen that $\operatorname{Bel}(B) \leq \operatorname{Pl}(B)$. A clear measure of ignorance about the opportunity $B$ and its completion $\bar{B}$ as the length of the interval $[\operatorname{Bel}(B), P l(B)]$ is allowed to be shown by a DST. This interval, which is called the belief interval (BI), can be depicted as the inaccuracy of the probability of opportunity $B$ [31] as well. In [14], Hong and Choi proposed an interval representation $\left[\mu_{A}\left(x_{j}\right), 1-v_{A}\left(x_{j}\right)\right]$ of IFS $A$ on $X$ instead of a pair $\left\langle\mu_{A}\left(x_{j}\right), v_{A}\left(x_{j}\right)\right\rangle$ in framework of MCDM problems.

The fact that the expression $\left[\mu_{A}\left(x_{j}\right), 1-v_{A}\left(x_{j}\right)\right]$ represents the authentic interval with its right bound being no smaller than the left one (due to the rule $\left.0 \leq \mu_{A}\left(x_{j}\right),+v_{A}\left(x_{j}\right) \leq 1\right)$ is the first obvious asset.

The consideration of the basic definition of the intuitionistic fuzzy sets theory with regards to the DST is the second asset.

The following definition was referred to in [31].

Definition 2. Let $X=\left\{x_{1}, x_{2}, \ldots, x_{n}\right\}$ be a universal finite set and $x_{j}$ be an element from $X$ described by functions $\mu_{A}\left(x_{j}\right), v_{A}\left(x_{j}\right)$, representing the membership 
and non-membership of element $x_{j} \in X$ to $A \subseteq X$, properly, such as $\mu_{A}: X \rightarrow[0,1]$, $x_{j} \in X \rightarrow \mu_{A}\left(x_{j}\right) \in[0,1]$ and $v_{A}: X \rightarrow[0,1], x_{j} \in X \rightarrow v_{A}\left(x_{j}\right) \in[0,1]$, and for any $x_{j} \in X, 0 \leq \mu_{A}\left(x_{j}\right)+v_{A}\left(x_{j}\right) \leq 1$. Intuitionistic fuzzy set $A$ in $X$ is an object of the following form: $A=\left\{\left\langle x_{j}, B I_{A}\left(x_{j}\right) \mid x_{j} \in X\right\rangle\right\}$, where $B I_{A}\left(x_{j}\right)=\left[\operatorname{Bel}_{A}\left(x_{j}\right), P l_{A}\left(x_{j}\right)\right]$ is a belief interval $\operatorname{Bel}_{A}\left(x_{j}\right)=\mu_{A}\left(x_{j}\right)$ and $P l_{A}\left(x_{j}\right)=1-v_{A}\left(x_{j}\right)$ are the belief and plausibility functions of $x_{j} \in X$ belonging to a set $A \subseteq X$.

When first examined, the definition of 1 expresses a simple re-definition of IFS as an interval fuzzy set, but the use of the DST semantic allows the increasing of the reliability of the calculations when dealing with operations on the IFVs and MCDM issues. Specifically, such an approach allows one to aggregate the local criteria determined by IFVs and the development of the MCDM method avoiding defuzzification during the time in which the local criteria and their weights are expressed by IFVs. Correspondingly, a final assessment in the form of the belief interval [31] is obtained.

\section{Operations on IFVs in framework of DST}

Two approaches of formulating the operation on belief intervals are suggested in paper [27]. The first approach is based on a probability interpretation of belief intervals. The second approach is based on a non-probability interpretation. In [27], the fact that the operations based on the non-probability interpretation of belief intervals have superior algebraic priorities to those based on the probability approach was proved. It is essential to indicate that arithmetic operators which have properties superior to algebraic operations done within the framework of the classical intuitionistic fuzzy sets theory are generated by each of the approaches. For this reason alone, operations defined in [27] based on the non-probability interpretation of belief intervals will be used by us.

Let $A=\left\langle\mu_{A}, v_{A}\right\rangle$ and $B=\left\langle\mu_{B}, v_{B}\right\rangle$ be the IFVs represented by belief intervals $B I(A)=[\operatorname{Bel}(A), P l(A)], \quad B I(B)=[\operatorname{Bel}(B), P l(B)], \quad$ where $\quad \operatorname{Bel}(A)=\mu_{A}$, $\operatorname{Pl}(A)=1-v_{A}$ and $\operatorname{Bel}(B)=\mu_{B}, \operatorname{Pl}(B)=1-v_{B}$, respectively. In this case, $\operatorname{Bel}(A)$ and $\operatorname{Pl}(A)$ are measures of belief and plausibility such as element $x_{j} \in X$ belongs to a set $A \subseteq X$. The belief interval $B I(A)=[\operatorname{Bel}(A), P l(A)]$ can be treated as an interval belonging a true power of ascertainment (argument, proposition, hypothesis etc.).

The additional and multiplication operators on belief intervals are shown in [27]. This becomes possible when we define an additional operator $\oplus_{B N P}$ of belief intervals as follows: 


$$
B I(A) \oplus_{B N P} B I(B)=\left[\frac{\operatorname{Bel}(A)+\operatorname{Bel}(B)}{2}, \frac{P l(A)+P l(B)}{2}\right] .
$$

So, if we have $n$ different ascertainments represented by belief intervals $B I\left(A_{i}\right)$, then their sum $\oplus_{B N P}$ can be defined as follows:

$$
B I\left(A_{1}\right) \oplus_{B N P} B I\left(A_{2}\right) \oplus_{B N P} \ldots \oplus_{B N P} B I\left(A_{n}\right)=\left[\frac{1}{n} \sum_{i=1}^{n} \operatorname{Bel}\left(A_{i}\right), \frac{1}{n} \sum_{i=1}^{n} \operatorname{Pl}\left(A_{i}\right)\right] .
$$

The multiplication operation $\bigotimes_{B N P}$ of belief intervals we can define as follows [27]:

$$
B I(A) \otimes_{B N P} B I(B)=[\operatorname{Bel}(A) \operatorname{Bel}(B), \operatorname{Pl}(A) \operatorname{Pl}(B)] .
$$

It can easily be seen that this multiplication operator is the same as the one used in the conventional interval arithmetic [37].

The scalar multiplication is defined in [27] as follows:

$$
\lambda B I(A)=[\lambda \operatorname{Bel}(A), \lambda P l(A)],
$$

where $\lambda$ is a real value, while $\lambda \in[0,1]$, because for $\lambda>1$ this operator does not always lead to the real belief intervals. This restriction is justified by the fact that we can define operations on belief intervals for MCDM problems, where $\lambda$ usually represents the weight of local criteria, which are smaller than one.

The exponentiation operation is defined in [27] as

$$
B I(A)^{\lambda}=\left[\operatorname{Bel}(A)^{\lambda}, \operatorname{Pl}(A)^{\lambda}\right],
$$

and it leads to a real belief interval for all $\lambda \geq 0$.

Using the conventional rules of interval arithmetic [38], we obtain:

$$
\begin{aligned}
B I(A)^{B I(B)}= & {\left[\min \left\{\operatorname{Bel}(A)^{\operatorname{Bel}(B)}, \operatorname{Pl}(A)^{\operatorname{Bel}(B)}, \operatorname{Bel}(A)^{P l(B)}, \operatorname{Pl}(A)^{P l(B)}\right\},\right.} \\
& \left.\max \left\{\operatorname{Bel}(A)^{\operatorname{Bel}(B)}, \operatorname{Pl}(A)^{\operatorname{Bel}(B)}, \operatorname{Bel}(A)^{P l(B)}, \operatorname{Pl}(A)^{P l(B)}\right\}\right] .
\end{aligned}
$$

Taking into account the properties of the belief intervals, we can lead these expressions to a following form [27]

$$
B I(A)^{B I(B)}=\left[\operatorname{Bel}(A)^{P l(B)}, P l(A)^{B e l(B)}\right] .
$$

The operators defined in that way have good algebraic properties (the same as in the case of the conventional theory of IFSs, see (5)-(10)). It is can be directly inferred from expressions (14)-(17): 


$$
\begin{gathered}
B I(A) \oplus_{B N P} B I(B)=B I(B) \oplus_{B N P} B I(A), \\
B I(A) \otimes_{B N P} B I(B)=B I(B) \otimes_{B N P} B I(A), \\
\left(B I(A) \otimes_{B N P} B I(B)\right)^{\lambda}=B I(A)^{\lambda} \otimes_{B N P} B I(B)^{\lambda}, \\
B I(A)^{\lambda_{1}} \otimes_{B N P} B I(A)^{\lambda_{2}}=B I(A)^{\lambda_{1}+\lambda_{2}}, \\
\lambda B I(A) \oplus_{B N P} \lambda B I(B)=\lambda\left(B I(A) \oplus_{B N P} B I(B)\right), \\
\lambda_{1} B I(A) \oplus_{B N P} \lambda_{2} B I(A)=\left(\lambda_{1}+\lambda_{2}\right) B I(A) .
\end{gathered}
$$

Using expressions (14) and (16) we get following Intuitionistic Weighted Arithmetic Mean (IWAM):

$$
\operatorname{IWAM}_{\text {DSTNP }}\left(A_{1}, A_{2}, \ldots, A_{n}\right)=\left[\frac{1}{n} \sum_{i=1}^{n} w_{i} \operatorname{Bel}_{A_{i}}, \frac{1}{n} \sum_{i=1}^{n} w_{i} P l_{A_{i}}\right] .
$$

This operator is not idempotent [27]. However, a small modification of (19) (multiplication by $n$ ) allows one to obtain an idempotent operator:

$$
\operatorname{IWAMI}_{\operatorname{DSTNP}}\left(A_{1}, A_{2}, \ldots, A_{n}\right)=\left[\sum_{i=1}^{n} w_{i} \operatorname{Bel}_{A_{i}}, \sum_{i=1}^{n} w_{i} P l_{A_{i}}\right] .
$$

The Intuitionistic Fuzzy Weighted Geometric operator $\left(I F W G_{D S T P}\right)$ obtained directly from (12) and (17) has the form [33]:

$$
I F W G_{D S T P}\left(A_{1}, A_{2}, \ldots, A_{n}\right)=\left[\prod_{i=1}^{n} B e l_{A_{i}}^{w_{i}}, \prod_{i=1}^{n} P l_{A_{i}}^{w_{i}}\right] .
$$

It is easy to see that the operator (21) is idempotent.

The Intuitionistic Fuzzy Weighted Geometric operator with weights presented by belief intervals $\left(I F W G B_{D S T P}\right) \quad B I_{i}=\left[B e l_{i}, P l_{i}\right], \quad i=1, \ldots, n$, obtained directly from (15) and (17) has the form [33]:

$$
\operatorname{IFWGB}_{D S T P}\left(A_{1}, A_{2}, \ldots, A_{n}\right)=\left[\prod_{i=1}^{n} \operatorname{Bel}_{A_{i}}^{P l_{i}}, \prod_{i=1}^{n} P l_{A_{i}}^{B e l_{i}}\right] .
$$

It showed in [33] that the result obtained by means of this operator has the form of belief intervals.

This operator is not idempotent. Of course, the idempotence of operator (21) is guaranteed by the normalization of weight value in the form of the real numbers, or $\sum_{i=1}^{n} w_{i}=1$. Given that in (22) weights are have a belief interval's form $B I_{i}=\left[B e l_{i}, P l_{i}\right]$. Observe that we have a problem with their normalization [27]. 
Using the proposed approach (15) and (20), we get IWAM in the case when the local criteria and their weights are IFVs.

Let $B I_{i}=\left[\mathrm{Bel}_{i}, P l_{i}\right], i=1, \ldots, n$, be belief intervals corresponding to the intuitionistic fuzzy weights of the local criteria $A_{i}, i=1, \ldots, n$ presented by belief intervals $B I\left(A_{i}\right)=\left[B e l_{A_{i}}, P l_{A_{i}}\right]$.

Then, from (15) and (19) we get [27]

$$
I W A M B_{D S T N P}\left(A_{1}, A_{2}, \ldots, A_{n}\right)=\frac{1}{n}\left[\sum_{i=1}^{n} \operatorname{Bel}_{i} \operatorname{Bel}_{A_{i}}, \sum_{i=1}^{n} P l_{i} P l_{A_{i}}\right] .
$$

The simple modification of foregoing operator (multiplying by $n$ ) allows one to obtain a more handy operator [27]:

$$
I W A M B_{D S T N P}\left(A_{1}, A_{2}, \ldots, A_{n}\right)=\left[\sum_{i=1}^{n} \operatorname{Bel}_{i} \operatorname{Bel}_{A_{i}}, \sum_{i=1}^{n} P l_{i} P l_{A_{i}}\right] .
$$

This operator is not idempotent. Of course, the idempotence of operator (24) is guaranteed by the normalization of weight value in the form of the real numbers, or $\sum_{i=1}^{n} w_{i}=1$, while in (21) weights have a belief interval's form $B I_{i}=\left[B e l_{i}, P l_{i}\right]$.

It seems that idempotance is notably important in MCDM issues among the basic properties of the aggregation operations (boundary conditions, monotonicity, continuity, symmetry, idempotance and others).

To conclude, it can be stated that the operators presented in the framework of the non-probability approach to the belief interval have their correspondents in the classical theory of IFSs [27].

It is important to remember that there is no definition of raising an IFV to intuitionistic fuzzy power in the classical intuitionistic fuzzy sets theory. So, considering the analysis of the raise to power converting IFSs to belief intervals, the following expression is obtained:

$$
A^{B}=\left\langle\mu_{A}^{1-v_{B}}, 1-\left(1-v_{A}\right)^{\mu_{B}}\right\rangle .
$$

Let us consider an example of $A^{B}$ calculating using the convert to belief intervals and expression (29).

Example 5. Let $A=\langle 0.78,0.2\rangle$ and $B=\langle 0.44,0.33\rangle$. Then $B I(A)=[0.78,0.8]$ and $B I(B)=[0.44,0.67]$, so $B I(A)^{B I(B)}=[0.8467,0.9065]$. Using expression (25), we get $A^{B}=\langle 0.8467,0.0935\rangle$. It is easy to see, that the results coincide qualitatively, and $B I\left(A^{B}\right)=[0.8467,0.9065]$. 
Similarly, their equivalent operator to $I F W G I_{D S T}$ is an operator presented in the expression (26):

$$
I F W G I=\left\langle\prod_{i=1}^{n} \mu_{A_{i}}{ }^{\left(1-v_{i}\right)}, 1-\prod_{i=1}^{n}\left(1-v_{A_{i}}\right)^{\mu_{i}}\right\rangle,
$$

\section{The properties of exponentiation operation realized by transformation to belief intervals and heuristic IFV method}

Let us consider the basic properties of exponential functions (18) and (25).

Let $A=\left\langle\mu_{A}, v_{A}\right\rangle, B=\left\langle\mu_{B}, v_{B}\right\rangle$ and $C=\left\langle\mu_{C}, v_{C}\right\rangle$ be IFV's. Then, belief intervals $B I(A)=\left[B e l_{A}, P l_{A}\right], B I(B)=\left[\mathrm{Bel}_{B}, P l_{B}\right]$ and $B I(C)=\left[B l_{C}, P l_{C}\right]$ are representation of IFVs.

It follows from (14)-(18) that

$$
B I(A)^{B I(B)+B I(C)}=B I(A)^{B I(B)} \otimes B I(A)^{B I(C)} .
$$

Proof. $B I(A)^{B I(B)} \otimes B I(A)^{B I(C)}=\left[B^{B e l_{A}}{ }^{P l_{B}}, P l_{A}{ }^{B e l_{B}}\right] \otimes\left[B e l_{A}{ }^{P l_{C}}, P l_{A}{ }^{B e l_{C}}\right]=$ $\left[\mathrm{Bel}_{A}{ }^{P l_{B}} \cdot \mathrm{Bel}_{A}{ }^{P l_{C}}, P l_{A}{ }^{B e l_{B}} \cdot P l_{A}{ }^{B e l_{C}}\right]=\left[\mathrm{Bel}_{A}{ }^{P l_{B}+P l_{C}}, P l_{A}{ }^{B e l_{B}+B e l_{C}}\right]=B I(A)^{B I(B)+B I(C)}$.

The next met property is

$$
\left(B I(A)^{B I(B)}\right)^{B I(C)}=B I(A)^{B I(B) \otimes B I(C)} .
$$

Proof. $\left(B I(A)^{B I(B)}\right)^{B I(C)}=\left(\left[\mathrm{Bel}_{A}{ }^{P l_{B}}, P l_{A}{ }^{B e l_{B}}\right]\right)^{\left[B e l_{C}, P l_{C}\right]}=\left[\left(\mathrm{Bel}_{A}{ }^{P l_{B}}\right)^{P l_{C}},\left(P l_{A}{ }^{B e l_{B}}\right)^{B e l_{C}}\right]=$ $\left[\mathrm{Bel}_{A}{ }^{P l_{B} \cdot P l_{C}}, P l_{A}{ }^{\mathrm{Bel}_{B} \cdot B e l_{C}}\right]=B I(A)^{B I(B) \cdot B I(C)}$.

So, in the same way, we get

$$
(B I(A) \otimes B I(B))^{B I(C)}=B I(A)^{B I(C)} \otimes B I(B)^{B I(C)} .
$$

Proof. $B I(A)^{B I(C)} \otimes B I(B)^{B I(C)}=\left[\mathrm{Bel}_{A}{ }^{P l_{C}}, P l_{A}{ }^{B e l_{C}}\right] \otimes\left[\mathrm{Bel}_{B}{ }^{P l_{C}}, P l_{B}{ }^{B e l_{C}}\right]=$ $\left[\mathrm{Bel}_{A}{ }^{P l_{C}} \mathrm{Bel}_{B}{ }^{P l_{C}}, P l_{A}{ }^{B e l_{C}} \mathrm{Pl}_{B}{ }^{B e l_{C}}\right]=\left[\left(\mathrm{Bel}_{A} \mathrm{Bel}_{B}\right)^{P l_{C}},\left(P l_{A} P l_{B}\right)^{B e l_{C}}\right]=(B I(A) \otimes B I(B))^{B I(C)}$.

However, the following properties are not met:

$$
\begin{gathered}
B I(A)^{B I(B)-B I(C)} \neq \frac{B I(A)^{B I(B)}}{B I(A)^{B I(C)}},\left(\frac{B I(A)}{B I(B)}\right)^{B I(C)} \neq \frac{B I(A)^{B I(C)}}{B I(B)^{B I(C)}}, \\
B I(A)^{-B I(B)} \neq \frac{1}{B I(A)^{B I(B)}} .
\end{gathered}
$$


In the case of expression (25) the following properties are met:

$$
\left(A^{B}\right)^{C}=A^{B \otimes C} .
$$

Proof. $\left(A^{B}\right)^{C}=\left\langle\mu_{A}{ }^{1-v_{B}}, 1-\left(1-v_{A}\right)^{\mu_{B}}\right\rangle^{C}=\left\langle\left(\mu_{A}{ }^{1-v_{B}}\right)^{1-v_{C}}, 1-\left(1-1-\left(1-v_{A}\right)^{\mu_{B}}\right)^{\mu_{C}}\right\rangle=$ $\left\langle\left(\mu_{A}{ }^{\left(1-v_{B}\right)\left(1-v_{C}\right)}, 1-\left(1-v_{A}\right)^{\mu_{B} \mu_{C}}\right\rangle=\left\langle\left(\mu_{A}{ }^{\left.1-v_{B}-v_{C}+v_{B} v_{C}\right)}, 1-\left(1-v_{A}\right)^{\mu_{B} \mu_{C}}\right\rangle=A^{B \otimes C}\right.\right.$.

The following property is also met

$$
(A \otimes B)^{C}=A^{C} \otimes B^{C} .
$$

Proof. $A^{C} \otimes B^{C}=\left\langle\mu_{A}{ }^{1-v_{C}}, 1-\left(1-v_{A}\right)^{\mu_{C}}\right\rangle \otimes\left\langle\mu_{B}{ }^{1-v_{C}}, 1-\left(1-v_{B}\right)^{\mu_{C}}\right\rangle=$ $\left\langle\mu_{A}{ }^{1-v_{C}} \cdot \mu_{B}{ }^{1-v_{C}}, 1-\left(1-v_{A}\right)^{\mu_{C}}+1-\left(1-v_{B}\right)^{\mu_{C}}-1+\left(1-v_{B}\right)^{\mu_{C}}+\right.$

$$
\left.+\left(1-v_{A}\right)^{\mu_{C}}-\left(1-v_{B}\right)^{\mu_{C}} \cdot\left(1-v_{A}\right)^{\mu_{C}}\right\rangle=
$$

$\left\langle\left(\mu_{A} \cdot \mu_{B}\right)^{1-v_{C}}, 1-\left(1-v_{B}\right)^{\mu_{C}} \cdot\left(1-v_{A}\right)^{\mu_{C}}\right\rangle=\left\langle\left(\mu_{A} \cdot \mu_{B}\right)^{1-v_{C}}, 1-\left(v_{A}+v_{B}-v_{A} \cdot v_{B}\right)^{\mu_{C}}\right\rangle=$ $=(A \otimes B)^{C}$.

Despite the fact that expression (25) is similar to (18) the property similar to (27) is not met:

$$
A^{B+C} \neq A^{B} \otimes A^{C}
$$

Due to the lack of a strict definition of subtraction of intuitionistic fuzzy sets, we have no way to conclusively determine whether the following properties are preserved: $A^{B-C}=\frac{A^{B}}{A^{C}},\left(\frac{A}{B}\right)^{C}=\frac{A^{C}}{B^{C}}, A^{-B}=\frac{1}{A^{B}}$.

\section{Conclusions}

The heuristic method to solve the problem of raising an intuitionistic fuzzy values to intuitionistic fuzzy power and Intuitionistic Fuzzy Weighted Geometric operator with weights presented by intuitionistic fuzzy values are proposed. The advantage of this heuristic method is lack of the requirement to convert intuitionistic fuzzy values. In addition, the properties of this method and method based on DST theory are proved. 


\section{References}

[1] Agrawal V.P., Kohli V., Gupta S., Computer aided robot selection: The multiple attribute decision making approach, International Journal of Production Research 1991, 29, 1629-1644.

[2] Alsina C., On a family of connectives for fuzzy sets, Fuzzy Sets and Systems 1985, 16, 231-235.

[3] Atanassov K.T., Intuitionistic fuzzy sets, Fuzzy Sets and Systems 1986, 20, 87-96.

[4] Atanassov K., New operations defined over the intuitionistic fuzzy sets, Fuzzy Sets and Systems 1994, 61, 137-142.

[5] Atanassov K., Pasi G., Yager R., Intuitionistic fuzzy interpretations of multi-person multicriteria decision making, Proc. of 2002 First International IEEE Symposium Intelligent Systems 1, 115-119.

[6] Atanassov K., Pasi G., Yager R., Atanassova V., Intuitionistic fuzzy group interpretations of multi-person multi-criteria decision making, Proc. of the Third Conference of the European Society for Fuzzy Logic and Technology EUSFLAT'2003, Zittau, 10-12 September (2003) 177-182.

[7] Cheng S., Chan C.W., Huang G.H., An integrated multi-criteria decision analysis and inexact mixed integer linear programming approach for solid waste management, Engineering Applications of Artificial Intelligence 2003, 16, 543-554.

[8] Choi D.Y., Oh K.W., Asa and its application to multi-criteria decision making, Fuzzy Sets and Systems 2000, 114, 89-102.

[9] Chu T.C., Facility location selection using fuzzy TOPSIS under group decisions, International Journal of Uncertainty, Fuzziness and Knowledge-Based Systems 2002a, 10, 687-701.

[10] Chu T.C., Selecting plant location via a fuzzy TOPSIS approach, The International Journal of Advanced Manufacturing Technology 2002b, 20, 859-864.

[11] Deluca A., Termini S., A definition of a nonprobabilistic entropy the of fuzzy sets theory, Information and Control 1972, 20, 301-312.

[12] Dempster A.P., Upper and lower probabilities induced by a muilti-valued mapping, Ann. Math. Stat. $1967,38,325-339$.

[13] Chen S.M., Tan J.M., Handling multicriteria fuzzy decision-maiking problemsbased on vague set theory, Fuzzy Sets and Systems 1994, 67, 163-172.

[14] Hong D.H., Choi C.-H., Multicriteria fuzzy decision-making problems based on vague set theory, Fuzzy Sets and Systems 2000, 114, 103-113.

[15] Li D.-F., Multiattribute decision making models and methods using intuitionistic fuzzy sets, Journal of Computer and System Sciences 2005, 70, 73-85.

[16] Li F., Lu A., Cai L., Methods of multi-criteria fuzzy decision making based on vague sets, Journal of Huazhong University of Science and Technology 2001, 29, 1-3 (in Chinese).

[17] Li F., Rao Y., Weighted methods of multi-criteria fuzzy decision making based on vague sets, Computer Science 2001, 28, 60-65 (in Chinese).

[18] Lin L., Yuan X.-H., Xia Z.-Q., Multicriteria fuzzy decision-making methods based on intuitionistic fuzzy sets, Journal of Computer and System Sciences 2007, 73, 84-88.

[19] Liu H.-W., Wang G.-J., Multi-criteria decision-making methods based on intuitionistic fuzzy sets, European Journal of Operational Research 2007, 179, 220-233.

[20] Pasi G., Yager Y., Atanassov K., Intuitionistic fuzzy graph interpretations of multi-person multicriteria decision making: Generalized net approach, Proceedings of 2004 Second International IEEE Conference Intelligent Systems 2004, 434-439.

[21] Pasi G., Atanassov K., Melo Pinto P., Yager R., Atanassova V., Multi-person multi-criteria decision making: Intuitionistic fuzzy approach and generalized net model, Proc. of the 10th ISPE International Conference on Concurrent Engineering "Advanced Design, Production and Management Systems", Madeira, 26-30 July 2003, 1073-1078. 
[22] Szmidt E., Kacprzyk J., Applications of intuitionistic fuzzy sets in decision making, Proc. Eighth Cong. EUSFLAT98, Pampelona 1998, 150-158.

[23] Szmidt E., Kacprzyk J., Group decision making under intuitionistic fuzzy preference relations, Proceedings of Seventh International Conference (IMPU98), Paris 1998, 172-178.

[24] Szmidt E., Kacprzyk J., Intuitionistic fuzzy sets in decision making, Notes IFS 1996, 2, 15-32.

[25] Szmidt E., Kacprzyk J., Remarks on some applications on intuitionistic fuzzy sets in decision making, Notes IFS 1996, 2, 22-31.

[26] Beliakov G., Bustince H., Goswami D.P., Mukherjee U.K., Pal N.R., On averaging operators for Atanassov's intuitionistic fuzzy sets, Information Sciences 2011, 182, 1116-1124.

[27] Dymova L., Sevastjanov P., The operations on intuitionistic fuzzy values in the framework of Dempster-Shafer theory, Knowledge-Based Systems 2012, 35, 132-143.

[28] Boran F.E., Cenc S., Kurt M., Akay D., A multi-criteria untuitionistic fuzzy group decision making for supplier selection with TOPSIS method, Expert Systems with Applications 2009, 36, 11363-11368.

[29] Xu Z., Xia M., Induced generalized intuitionistic fuzzy operators, Knowledge-Based Systems 2011, 24, 197-209.

[30] Yang W., Chen Z.,The quasi-arithmetic intuitionistic fuzzy OWA operators, Knowledge-Based Syst. 2011

[31] Dymova L., Sevastjanov P., An interpretation of intuitionistic fuzzy sets in terms of evidence theory, Decision making aspect, Knowledge-Based Systems 2010, 23, 772-782.

[32] Hinde C.J., Patching R.S., McCoy S.A., Inconsistent Intuitionistic Fuzzy Sets and Mass Assignment, EXIT, 2007.

[33] Hinde C.J., Patching R.S., McCoy S.A., Semantic transfer and contradictory evidence in intuitionistic fuzzy sets, Proc. of 2008 IEEE International Conference on Fuzzy Systems, 2008, 2095-2102.

[34] Dymova L., Sevastjanov P., Two-criteria method for comparing real-valued and interval-valued intuitionistic fuzzy values, Knowledge-Based Systems 2013, 45, 166-173.

[35] Dey S.K., Biswas R., Roy A.R., Some operations on intuitionistic fuzzy sets, Fuzzy Sets and Systems 2000, 114, 477-484.

[36] Xu Z., Intuitionistic preference relations and their applications in group decision making, Information Sciences 2007, 177, 2363-2379.

[37] Shafer G., A Mathematical Theory of Evidence, Princeton University Press, Princeton 1976.

[38] Moore R.E., Interval Analysis, N.J. Prentice-Hall, Englewood Cliffs 1966. 